\title{
A chemical sensor based on a photonic-crystal L3 nanocavity defined into a silicon-nitride membrane
}

\author{
Kieran Deasy ${ }^{\mathrm{a}, \dagger}$, Khalid Sediq ${ }^{\mathrm{a}}$, Stuart Brittle ${ }^{\mathrm{a}}$, Tao Wang ${ }^{\mathrm{a}}$, Frank Davis ${ }^{\mathrm{b}}$, Tim \\ Richardson ${ }^{\text {a }}$ and David Lidzey ${ }^{\mathrm{a}, *}$ \\ a. Department of Physics and Astronomy, University of Sheffield, Sheffield, S3 7RH, \\ United Kingdom \\ b. Cranfield Biotechnology Centre,School of Engineering, Cranfield MK43 OAL, \\ United Kingdom \\ $\dagger$ : Current address: Light-Matter Interactions Unit, OIST Graduate University, 1919-1 \\ Tancha, Onna-son, Okinawa 904-0495, Japan \\ * Electronic mail: d.g.lidzey@sheffield.ac.uk
}

\begin{abstract}
The optical properties of a silicon-nitride based L3 optical nanocavity are explored as a chemical sensor. It is shown that by adjusting the thickness of an ultra-thin Lumogen Red film deposited onto the nanocavity surface, the fundamental optical mode undergoes a progressive red-shift as the layer-thickness increases, with the cavity being able to detect the presence of a single molecular monolayer. The optical properties of a nanocavity whose surface is coated with a thin layer of a porphyrin-based polymer are also explored. On exposure of the cavity to an acidic-vapour, it is shown that changes in the optical properties of the porphyrin-film (thickness and refractive index) can be detected through a reversible shift in the cavity mode wavelength. Such effects are described using a finite difference time-domain model.
\end{abstract}




\section{Introduction}

Photonic crystals [1] $(\mathrm{PhC})$ are periodic optical structures that possess a photonic bandgap and can therefore be used to restrict the propagation of light. By creating a physical defect into a $\mathrm{PhC}$, an optical nanocavity can be created that can be used to confine light in 3 dimensions [2-4]. Such structures can have high cavity Q-factors and small mode volumes $(\mathrm{V})$, with the resultant high $\mathrm{Q} / \mathrm{V}$ values providing a method to enhance radiative rates of emissive materials placed within the cavity via the Purcell effect. Nanocavities thus provide a test-bed to study fundamental processes $[5,6]$ and permit the realization of optoelectronic devices [7].

There is now however significant interest in the use of PhC-based optical cavities as optical sensors. Here, the small modal volumes of a nanocavity combined with a strongly localized electromagnetic field provide an effective means to detect small changes in the local dielectric constant of the surrounding medium [8,9], or the binding of specific biological materials [10-15] to the cavity surface through shifts in the wavelength of the cavity modes. For a full review of the literature on this subject, we refer the reader to a recent review [16]. A number of such biosensor devices utilize a silicon on insulator (SOI) chip as the medium into which the $\mathrm{PhC}$ and cavity is defined. Here the combination of a high refractive index silicon layer $(n=3.5)$ positioned above a low index silicon oxide cladding $(n=1.5)$ provides tight optical confinement in the silicon, creating an effective waveguide structure for light around 1.3 to $1.5 \mu \mathrm{m}$.

Whilst SOI-based structures clearly offer the advantages of effective optical confinement and a well-developed platform for lithography, their operation at infra-red based wavelengths make them less sensitive to refractive-index changes resulting from the selective binding of many organic (carbon-based) materials, as organic materials often have a higher refractive index at UV or visible wavelengths (i.e. close to an electronic transition) [17]. It is clear however that the increased sensitivity to large changes in refractive index obtained by sensing materials at visible wavelengths will come at the price of reduced cavity Q-factor, as the dielectric materials used to construct the cavity have lower refractive index and thus confine light less effectively [18]. Furthermore, the reduced refractive index of the cavity dielectric will also necessitate such cavities to operate in air rather than in an aqueous environment. This is in contrast to systems based on SOI, where the high refractive index contrast between silicon and water permits the formation of photonic crystals having an optical band-gap. This will clearly limit the type of materials that could be sensed by a nanocavity operating at visible wavelengths to those carried by air. Nevertheless, there are plenty of applications for air-based detectors, such as the detection of air-borne pollutants, pathogens or explosive materials etc. 


\section{Results and discussion}

In this paper, we explore the use of low Q-factor nanocavities built using the material silicon nitride ( $\mathrm{SiN}$ ) as optical sensors. We demonstrate that such cavities can be used to detect the surface deposition of a molecular monolayer - corresponding to the binding of as little as $0.35 \mathrm{fg}$ of material to the cavity surface. We then demonstrate that by incorporating a thin functionalized polymer layer at the surface of a SiN L3 nanocavity, small changes in cavity volume can be detected as the polymer swells in response to exposure to an acidic vapor. We argue that such SiN-based structures show promise as chemical and biochemical sensors having a sensitivity that is in many cases comparable to similar structures constructed using SOI.

The cavities explored were fabricated from $\mathrm{SiN}$; a dielectric material having a band-gap between 2.43 and $4.74 \mathrm{eV}$ (dependent on growth conditions) [19] and a refractive index of $n \sim$ 2.1. There has been significant interest in the use of $\mathrm{SiN}$ as a medium in which to fabricate optical nanocavities [20-25] including the demonstration of a $\mathrm{SiN}$ nanobeam [26] cavity having a Q-factor of $\sim 55,000$. Indeed, an H1 SiN cavity (consisting of a single hole missing from a photonic crystal composed of triangular lattice of holes) has also been chemically functionalized to bind fluorescently labeled antibodies or DNA [27]. Here, the SiN optical nanocavities that we have explored are based on a so-called L3 structure, where three holes are 'missing' from a photonic crystal. A scanning electron microscope image of a typical nanocavity is shown in Figure 1(a). The nanocavities were fabricated on a free standing 200 $\mathrm{nm}$ thick SiN membrane (supplied by Silson Ltd.) and were based on a photonic crystal comprising a triangular lattice of holes having a lattice constant $a=260 \mathrm{~nm}$. Our previous work [22] has demonstrated that such holes penetrate the entire thickness of the membrane, and - due to the reactive-ion etching technique used to transfer the electron-beam written pattern to the $\mathrm{SiN}$ - have a low cone angle of $2.5^{\circ}$.

The cavities explored were designed using a finite difference time domain (FDTD) engine (Crystalwave software supplied by Photon Design Ltd.), and were constructed such that the fundamental cavity mode was located at approximately $650 \mathrm{~nm}$ [22]. Two different cavity designs were explored; cavity (1) was defined into a PhC having a hole diameter of $\mathrm{d}=$ $0.6 a$, with the holes at the end of the cavity long-axis shifted outwards by $\mathrm{S}=0.24 a$, see Figure 1(d). Such techniques have been shown to reduce leaky modes from the cavity and thus improve cavity Q-factor [22], with the structures explored here having a Q-factor of 1,500. In cavity (2) the four holes along the long axis of the cavity had a hole diameter of $d=$ $0.5 a$, with the first three holes at either end of the cavity having a diameter of $0.4 a$ and shifted by $\mathrm{S} 1=0.22 a, \mathrm{~S} 2=0.025 a$ and $\mathrm{S} 3=0.18 a$ respectively (as shown in Figure $1(\mathrm{e})$ ). Here, the cavity Q-factor was lower at $\sim 1,000$. We plot the distribution of the fundamental cavity mode as calculated using the FDTD model in Figures 1(b) \& (c). 
To explore the response of a nanocavity to a precise change in the thickness of a thin-film deposited onto a cavity surface, we have used the Langmuir-Schaefer (LS) technique to deposit sequential monolayers of the molecular dye Lumogen Red onto the surface of cavity (1) with very high precision. We have determined the area per molecule of the LS deposited films from an isotherm recorded during the compression of the Lumogen Red monolayers on the water sub-phase (data not shown). Using this, we calculate the area subtended by each molecule at the water surface to be $1.5 \mathrm{~nm}^{2}$. Spectroscopic ellipsometry was also used to determine the thickness of a single Lumogen Red monolayer and the refractive index as an input to our modelling, and was found to be $1.2 \mathrm{~nm}$ and 1.65 respectively.

We have previously explored the use of Lumogen Red in optical nanocavities [21, 22] and micropillars [28]. Lumogen Red is highly photostable, and absorbs light efficiently at wavelengths below $600 \mathrm{~nm}$, and emits fluorescence with high quantum efficiency from 575 $\mathrm{nm}$ to $750 \mathrm{~nm}$, with a maximum at $600 \mathrm{~nm}$ [28]. In our experiments we use Lumogen Red as it can be easily deposited on a surface with monomolecular precision, with its strong, red-fluorescence emission permitting us to effectively study the effect of a surface molecular layer on the optical mode wavelength of a SiN L3 nanocavity. Like many organic materials, Lumogen Red is expected to have a relatively low relative permittivity and thus acts as a reasonable model for a biomolecule [29]. Note however, a practical implementation of our cavities as a sensor would not include Lumogen Red. Rather (as we demonstrate below), this can be achieved by coating the cavity surface with a material whose optical properties can be modified in response to chemical species present in their surrounding environment.

We have studied the photoluminescence (PL) emission from nanocavities following excitation using a CW laser $(\lambda=442 \mathrm{~nm})$. All measurements were made in air. Figure 2(a) shows a schematic of our spectroscopy setup, with further details about our methods described in the experimental methods section. By setting the spectrometer grating to zeroth-order, we can also use our system to create a direct, real-space fluorescence image of the nanocavity surface. This is shown in Figure 2(b) and (c) for a nanocavity before and after being coated by a series of Lumogen-Red molecular monolayers. It can be seen that a bright, emissive spot is detected in the centre of the image that corresponds to the nanocavity region. The edge of the photonic crystal region is similarly bright, with emission resulting from enhanced optical scattering. In all cases, we use this image to define the region on the CCD detector over which emission is recorded, allowing us to probe the optical properties of the nanocavity whilst limiting our sensitivity to its surrounding (non-cavity) regions.

Figure 3 shows a series of PL spectra recorded from nanocavity (cavity 1) coated with an increasing number of molecular monolayers of Lumogen Red. Here, we focus on the emission around the wavelength of the fundamental cavity mode at $650.5 \mathrm{~nm}$. Note, even in a cavity with no Lumogen Red present, we still detect luminescence from the cavity mode; an 
effect that originates from the weak intrinsic fluorescence of silicon nitride. It can be seen that the wavelength of the fundamental mode undergoes a red-shift as the number of monolayers increases, going from $650.8 \mathrm{~nm}$ when the cavity is coated with a single monolayer to $654.0 \mathrm{~nm}$ after 16 monolayers have been deposited.

We plot the fluorescence intensity emitted from the nanocavity as a function of the number of LS deposited films as shown in Figure 4(a), with the peak emission wavelength shown in Figure 4(b). Here, the fluorescence intensity (wavelength) was obtained from the area (peak) of a Lorentzian function fit to each of the features shown in Figure 3 after an emission background had been subtracted to account for the intrinsic fluorescence of the SiN. It can be seen that the luminescence emission is approximately linear as a function of the Lumogen Red layer thickness. To understand the origin of this dependence, we have used FDTD modeling (not shown) to calculate the average value of the electromagnetic field throughout the organic layer. Our model suggests that the average electromagnetic field intensity within this layer is approximately constant (to within $\pm 12 \%$ ) over the range of film thicknesses explored here. This indicates that no significant changes in the coupling efficiency of the fluorescence emission to the cavity mode can be expected. We conclude therefore that the linear increase in luminescence observed as a function of Lumogen Red layer thickness simply results from the fact that there is progressively more material at the nanocavity surface whose fluorescence is able to couple to the confined optical field.

It can be seen in Figure 4(b) that the cavity mode undergoes a red-shift as Lumogen Red monolayers are deposited onto the cavity surface. Such a change in cavity mode wavelength results from a change in the overall thickness and effective refractive index of the nanocavity and surrounding photonic crystal. We note that similar effects have been previously observed following the deposition of materials such as xenon [30] or $\mathrm{Al}_{2} \mathrm{O}_{3}$ [31] onto a nanocavity surface or by using reversible refractive index changes in photochromic films to tune nanocavity wavelength [32]. We can model the effect of the organic surface layer on the optical mode-structure of our cavity using an FDTD model. Using this, we calculate the cavity mode wavelength as a function of the number of Lumogen Red monolayers using measured values of thickness $(1.2 \mathrm{~nm})$ and refractive index $(\mathrm{n}=1.65)$ as input as shown in Figure 4(b). This is plotted against measured cavity mode wavelength, with a good agreement achieved between the model and the experimental data. Note, that as the thickness of the surface layer increases beyond a certain critical level (around $50 \mathrm{~nm}$ ), the cavity mode starts to broaden appreciably. For example, our FDTD model indicates that the FWHM of a cavity coated with a $15 \mathrm{~nm}$ thick layer having a refractive index of $\sim 1.80$ increases from 1.04 to $3.44 \mathrm{~nm}$ as the thickness of this surface layer is increased to $60 \mathrm{~nm}$. Such a broadening will clearly act to limit the effectiveness by which subtle changes in film thickness can be followed, and thus SiN L3 cavity are most appropriately used in conjunction with thin $(<50 \mathrm{~nm})$ organic films. 
The ability of the silicon-nitride based nanocavities to act as a simple optical sensor is evident. The width of the cavity mode resonance in our cavities is $0.45 \mathrm{~nm}$, and if we arbitrarily define a limit of detection sensitivity as being half the cavity mode linewidth, then our structures should be sensitive to a mode shift of $0.225 \mathrm{~nm}$. Using a simple linear fit, we determine that a spectral shift of $0.22 \mathrm{~nm}$ occurs following the deposition of a single Lumogen Red monolayer. This shift coincides with limit of detectability, indicating that our cavities can be used to detect films having an average thickness of a single monolayer. We estimate the surface area of our cavity to be $3 \times 10^{5} \mathrm{~nm}^{2}$. We define this area as the surface-area enclosed within the 10 holes that surround the cavity medium (defined by a line that runs through the centre of each of these circles). If we now estimate that each Lumogen Red molecule subtends an area of $1.5 \mathrm{~nm}^{2}$, we estimate that our cavity has a detection sensitivity of $\sim 0.35 \mathrm{fg}$.

To explore the application of our structures as chemical sensors we have explored the optical properties of L3 nanocavities coated with a thin layer of a copolymer of tetraaminophenyl porphyrin and diaminodurene with the fluorinated dianydride (with $\mathrm{x}=$ $5 \%$ ). The chemical structure of this polymer is shown in Figure 5(a), which for simplicity we refer to as PP1. To combine the PP1 polymer with our nanocavities, we have again used the LS technique. Fluorinated-polyimide polymers have previously been explored for their sensing ability in simple thin-film format, with changes in spectral-position and intensity of fluorescence used to detect water, ethanol and isopropanol vapour [33]. Here, the incorporation of the porphyrin moiety gives additional benefits from both the porphyrin ring itself and its amine substituents. Amines are readily protonated by acidic materials such as trifluoroacetic acid (TFA). The repulsion between the resultant ionised species will cause the polymer to swell as well as changing its refractive index. In this work, we use the fact that on exposure to an acid vapour, the PP1 polymer swells and increases in refractive index, with both effects anticipated to red-shift the cavity mode wavelength. We can in fact follow this process in a non-cavity (control) PP1 film coated on a silicon wafer using spectroscopic ellipsometry, with measurements recorded every $1.7 \mathrm{~s}$ as shown in in Figure 5(b). Here it can be seen that over a TFA exposure period of $330 \mathrm{~s}$ (with exposure starting at $t=30 \mathrm{~s}$ ), the PP1 film expands by around $5 \mathrm{~nm}$, with its refractive index increasing from 1.779 to 1.798 (refractive index data not shown). It is apparent that the rate at which the film thickness slows as a function of time following the initial exposure. Previous work suggests that changes in the optical properties of these films on exposure to a test gas saturate in a time-scale between 2 and 10 minutes $[34,35]$. We estimate that full swelling saturation time for a PP1 film is around 5 minutes, although we obtain a measurable response from our sensor after a TFA exposure of less than 30 seconds.

In figure 5(c), we plot PL emission following laser excitation for an uncoated nanocavity and a nanocavity that has been coated with 5 monolayers of PP1. Before coating 
the nanocavity, the fundamental cavity mode is located at $657.3 \mathrm{~nm}$. After the LS deposition of the PP1, a strong peak is seen at $663.5 \mathrm{~nm}$ that originates from PP1 emission that is coupled into the fundamental cavity mode. In the same plot, we also show PL emission from the same cavity after it has been exposed to a TFA vapor for 3 minutes. Note that the local TFA concentration during this exposure process was neither controlled nor measured. It can be seen that this exposure results in a red-shift of the cavity mode wavelength by $(1.3 \pm 0.3)$ $\mathrm{nm}$ to $664.8 \mathrm{~nm}$. This change most probably results from a change in molecular conformation of the porphyrin polymer as a result of protonation of the amino groups. We have found that this red-shift is reversible; by heating the structure to $70^{\circ} \mathrm{C}$ in air for 5 minutes and then cooling it to room-temperature, the cavity mode blue-shifts to $663.3 \mathrm{~nm}$. This process effectively 're-sets' the optical sensor by raising the PP1 above its glass transition temperature and facilitating the escape of trapped TFA. This then results in a re-ordering of PP1 molecular structure to its equilibrium conformation. Note that previous work has shown that this process is repeatable over at least 10 cycles [36] and that porphyrin polymers have a high degree of stability and that degradation is insignificant over the period of an entire year [37].

We can again use FDTD calculations to confirm the effect of the swelling of the PP1 polymer on the optical properties of the cavity. As input into our model, we assume an L3 structure based on cavity design 2 as described earlier, with the cavity defined into a PhC made from a dielectric having a refractive index of 2.05. Onto this structure, we model a layer of various thickness having a refractive index of 1.779 (approximating the PP1 layer) to determine the shift in the cavity mode wavelength.

The results of our model are shown in Figure 5(d), where we plot the change in the simulated cavity mode wavelength as a function of surface layer thickness. Again, we find that the cavity mode wavelength undergoes a linear shift as the surface layer thickness increases. Experimentally, we determine a shift in cavity mode wavelength of $(1.3 \pm 0.3) \mathrm{nm}$, which our FDTD calculation indicates equates to a change in PP1 thickness of $3.25 \mathrm{~nm}$. Using the ellipsometry measurements shown in Figure 5(b) it can be seen that the PP1 film swells by $3.8 \mathrm{~nm}$ after a 3 minute TFA exposure; a value in good agreement with the thickness change determined on the basis of the shift in the cavity-mode. Any difference between the calculated and measured values is most easily accounted for by small changes in the refractive index of the PP1 layer that occur as it swells that are not taken account in our calculations. Again using the half-width of the cavity mode linewidth as a limit for the detection resolution, we estimate that we will be able to detect a minimum change in the thickness of the PP1 surface layer of $(1.4 \pm 0.3) \mathrm{nm}$. Note that our model indicates that if we instead fix the thickness of the PPI surface layer at $15 \mathrm{~nm}$, we obtain a wavelength shift of $\Delta \lambda$ $=0.34 \mathrm{~nm}$ for a change in refractive index of $\Delta n=0.02$.

For completeness, we have used our FDTD model to simulate the effect of similar 
changes in polymer thickness for a cavity in which the optical mode was located around 1500 $\mathrm{nm}$; a wavelength at which the refractive index of the polymer is significantly reduced. In our calculations, we assume a refractive index for the cavity medium of $n=3.0$, with the polymer having a refractive index of $n=1.54$ (a value typical of many polymeric materials around this wavelength [38]. Here, we find a wavelength-shift for the cavity mode $(\Delta \lambda)$ as function of change in the polymer thickness $(\Delta d)$ of $\Delta \lambda / \Delta d \approx 0.1$. This value is significantly smaller than the wavelength shift seen in our SiN cavities (see figure $5(\mathrm{~d})$ ), where $\Delta \lambda / \Delta d=0.4$, a result we partly ascribe to the larger refractive index of the polymer around $660 \mathrm{~nm}$.

\section{Conclusions}

In summary, we have shown that a SiN based nanocavity can be used to detect the deposition of a molecular monolayer onto its surface, and the swelling of a nanometer-thick porphyrin-based polymer film on a nanocavity surface as it is exposed to an acidic vapour. Despite the fact that the nanocavity has a relatively low Q-factor, we find that it has comparable sensitivity (in terms of its ability to detect the binding of fg quantities of material bound to the cavity surface) as other SOI-based nanocavity systems that operate at near infra-red wavelengths [16]. We believe that this sensitivity comes from the fact that our cavities operate at visible wavelengths $(\sim 650 \mathrm{~nm})$ where refractive index changes of the materials that we seek to detect is relatively larger. We also demonstrated that by coating the surface of the cavity with a thin-film of a porphyrin-based polymer, we could detect a swelling of the polymer through a shift in the cavity mode wavelength as the structure was exposed to an acidic vapor. Our work demonstrates therefore the applicability of silicon-nitride based photonic crystal nanocavities as simple optically-based chemical sensors. Indeed, we note that porphyrin based materials have been used to selectively recognize a variety of different materials, including dopamine [39] and mercury(II) [40]. Sensors utilizing other polymers that undergo swelling can be envisaged to detect vapors such as chloroform [41] or ammonia [42]. To increase the sensitivity of our sensors further, we believe it will be possible to coat or pattern a cavity surface with a molecular imprinted polymer (MIP) [43]. This technique can be used to imprint a surface thereby allowing it to selectively recognize and locally concentrate a particular molecular species. We expect that by developing techniques to selectively pattern different MIP films or functionalized organic materials on a nanocavity surface, it will be possible to create a series of cavities integrated onto a single substrate that can selectively detect a range of target materials, permitting such structures to form the basis of an artificial nose.

\section{Experimental methods}

To fabricate cavities into the silicon nitride membrane, a thin layer of PMMA was spin 
cast onto the surface of the membrane and then patterned using electron beam lithography. This was then developed using a (1:3) solution of MIBK:IPA and the pattern transferred to the silicon nitride via inductively coupled plasma etching with $\mathrm{CHF}_{3}$, with careful control over etch rates ensuring that the photonic crystal had near-vertical side-walls. The structure was then placed in an oxygen plasma asher to remove any remaining PMMA on the cavity surface.

To deposit the LS films onto the nanocavities, the PP1 and Lumogen Red molecules were dissolved in chloroform and spread on a clean water (Elga PURELab Option $>15 \mathrm{M} \Omega \mathrm{cm}$ ) subphase surface in a NIMA BAM Langmuir trough. After evaporation of the chloroform solvent, the film was compressed to a surface pressure of $15 \mathrm{mN} / \mathrm{m}$ whilst a LS deposition was performed. Here the substrate was held almost parallel to the surface of the water and was then lowered until the entire surface of the substrate made contact with the liquid. It was then retracted vertically, with a molecular monolayer being transferred to the substrate. This process was repeated sequentially, with as many as 16 monolayers being deposited onto the nanocavity. Note, that as the substrates are retracted, they were deliberately tilted such that any remaining water drops on the surface could easily run off and be collected in one corner.

To explore the optical properties of our cavities, we have generated fluorescence emission using a $442 \mathrm{~nm} \mathrm{HeCd}$ laser focused onto the sample through a 50X objective lens (0.55 NA), see Figure 2(a). PL emission was collected through the same lens and then imaged into a liquid-nitrogen cooled CCD detector, see Figure 2(b) \& (c). The laser was focused to a $2.5 \mu \mathrm{m}$ diameter spot, with the flux density at the surface being approximately $2.5 \times 10^{5}$ $\mathrm{Wcm}^{-2}$. All measurements were done in air. We estimate the spectral resolution of our spectrometer to be $0.7 \AA$. A polarizer was placed in front of the spectrometer allowing us to identify and probe the optical properties of the fundamental cavity mode. For all samples studied, a similar excitation and detection protocol was used, with fluorescence recorded during a single exposure lasting 10 seconds.

The optical properties of PP1 and Lumogen Red films were explored using a M2000v, J.A. Woollam Co. ellipsometer, which permitted us to determine film thickness and refractive index employing a Cauchy model to fit $\Psi$ (the ratio of the amplitude of the incident and reflected light beams) and $\Delta$ (the ratio of the phase lag of the incident and reflected light beams). Spectroscopic ellipsometry was also used to characterise the change in refractive index and thickness as the film was exposed to a TFA vapour. Here a cotton bud was dipped into a TFA solution and held in close proximity to the PP1 film. A similar procedure was also used to test the response of the PP1 nanosensor nanocavity.

\section{Acknowledgements}

This research was funded by the European Commission via the Marie Curie Initial 
Training Network ICARUS under Grant No. FP7-PEOPLE-ITN-2008-237900 and by the UK EPSRC via research grant EP/I028641/1. K.N.S. thanks the Kurdistan Regional Government for the provision of a research studentship. The molecular material Lumogen Red was kindly provided by ColorFlex GmbH. Tim Richardson died after a short illness during the course of this work, and so this manuscript is dedicated to his memory.

\section{References}

1. J. D. Joannopoulos, S. G. Johnson, J. N. Winn and R. D. Meade, Photonic Crystals: Molding the flow of light (Princeton University Press, Princeton 2008).

2. Y. Akahane, T. Asano, B. S. Song and S. Noda, Nature, 2003, 425, 944.

3. Y. Akahane, T. Asano, B. S. Song and S. Noda, Optics Express, 2005, 13, 1202.

4. A. R. A. Chalcraft, S. Lam, D. O’Brien, T. F. Krauss, M. Sahin, D. Szymanski, D. Sanvitto, R. Oulton, M. S. Skolnick, A. M. Fox, D. M. Whittaker, H. Y. Liu and M. Hopkinson, Appl. Phys. Lett.,2007,90, 241117.

5. T. Yoshie, A. Scherer, J. Hendrickson, G. Khitrova, H. M. Gibbs, G. Rupper, C. Ell, O. B. Shchekin and D. G. Deppe, , Nature,2004, 432, 200.

6. M. Barth, J. Kouba, J. Stingl, B. Lochel and O. Benson, Optics Express,2007,15,17231.

7. H.-G. Park, S.-H. Kim, S.-H. Kwon, Y.-G. Ju, J.-K. Yang, J.-H. Baek, S.-B. Kim and Y.-H. Lee, Science, 2004, 305, 1444-1447.

8. D. F. Dorfner, T. Hurlimann, T. Zabel, L. H. Frandsen, G. Abstreiter and J. J. Finley, Appl. Phys. Lett., 2008, 93, 181103.

9. C. Kang, C. T. Phare, Y. A. Vlasov, S. Assefa and S. M. Weiss, Optics Express , 2010, 18, 27930-27937.

10. S. Mandal, J. M. Goddard and D. Erickson, Lab Chip, 2009, 9, 2924-2932.

11. D. Dorfner, T. Zabel, T. Hurlimanna, N. Haukea, L. Frandsenb, U. Ranta, G. Abstreitera and J. Finley, Biosensors and Bioelectronics,2009,24,3688-3692.

12. M. Lee and P. M. Fauchet, Optics Express, 2007,15,4530 - 4535.

13. M.G. Scullion, A. Di Falco and T.F. Krauss, Biosensors and Bioelectronics, 2011, 27,101- 105 .

14. W.-C. Lai, S. Chakravarty, Y. Zou and R.T. Chen, Optics Letters, 2012, 37, 1208-1210.

15. S. Pal, E. Guillermain, R. Sriram, B.L. Miller and P. M. Fauchet, Biosensors and Bioelectronics,2011,26, 4024-4031.

16. S. Pal, P.M. Fauchet, B.L. Miller, Anal. Chem., 2012, 84, 8900-8908.

17. M. Fox, Optical Properties of Solids (Oxford University Press Inc, New York 2001).

18. A. M. Adawi, A. R. A. Chalcraft, D. M. Whittaker and D. G. Lidzey, Optics Express, 2007, 15, 14299.

19. S.V. Deshpande, E. Gulari, S.W. Brown and S.C. Rand, J. Appl. 
Phys.,1995,77,6536-6541.

20. M. M. Murshidy, A. M. Adawi, P. W. Fry, D. M. Whittaker and D. G. Lidzey, J. Opt. Soc. Am. B, 2010, 27, 215.

21. M. M. Murshidy, A. M. Adawi, P. W. Fry and D. G. Lidzey, Appl. Phys. Lett., 2010, 97, 153303.

22. A. M. Adawi, M. M. Murshidy, P. W. Fry and D. G. Lidzey, ACS Nano, 2010, 4, 3039.

23. A. M. Adawi and D. G. Lidzey, Mater. Sci. Eng. B, 2008, 149, 266-269.

24. M. Barth, N. Nüsse, J. Stingl, B. Löchel and Oliver Benson, Appl. Phys. Lett., 2008, 93, 021112.

25. M. Markarova, J. Vuckovic, H. Sanda and Y. Nishi, Appl. Phys. Lett., 2006, 89, 221101.

26. M. Khan, T. Babinec, M. W. McCutcheon, P. Deotare and M. Loncar, Optics Letters, 2011, 36, 421-423.

27. F. Pisanello, L. Martiradonna, A. Qualtieri, T. Stomeo, M. Grande, P. P. Pompa, R. Cingolani, A. Bramati and M. De Vittorio, Photonics and Nanostructures - Fundamentals and Applications, 2012, 10, 319-324.

28. A. M. Adawi, A. Cadby, L. G. Connolly, W.-C. Hung, R. Dean, A. Tahraoui, A. M. Fox, A. G. Cullis, D. Sanvitto, M. S. Skolnick, D. G. Lidzey, Adv. Mater., 2006, 18, 742-747.

29. M. K. Gilson and B. H. Honig, Biopolymers, 1986, 25, 2097-2119.

30. S. Mosor, J. Hendrickson, B. C. Richards, J. Sweet, G. Khitrova, H. M. Gibbs, T. Yoshie, A. Scherer, O. B. Shchekin and D. G. Deppe, Appl. Phys. Lett., 2005, 87, 141105.

31. S. Kiravittaya, H. S. Lee, L. Balet, L. H. Li, M. Francardi, A. Gerardino, A. Fiore, A. Rastelli and O. G. Schmidt, J. Appl. Phys., 2011, 109, 053115.

32. D. Sridharan, R. Bose, H. Kim, G. S. Solomon and E. Waks, Optics Express, 2011, 19, 5551.

33. S. Carturan, A. Quaranta, E. Negro, M. Tonezzer, M. Bonafini, G. Maggioni, G. Della Mea, Sensors and Actuators B, 2006, 118, 393-398.

34. S. Brittle, T. H. Richardson, A. D. F. Dunbar, S. Turega, C. A. Hunter, J. Phys. Chem. B, 2008, 112, 11278-11283.

35. A. F. Gutiérrez, S. Brittle, T. H. Richardson, A. Dunbar, Sensors and Actuators B: Chemical, 2014, 202, 854-860.

36. T. H. Richardson, C. M. Dooling, L. T. Jones, R. A. Brook, Advances in Colloid and Interface Science, 2005, 116, 81-96.

37. J. M. Pedrosa, C. M. Dooling, T. H. Richardson, R. K. Hyde, C. A. Hunter, M. T. Martin, L. Camacho, J. Mater. Chem., 2002, 12, 2659-2664.

38. Y.-G. Zhao, W.-K. Lu, Y. Ma, S.-S. Kim, S. T. Ho and T. J. Marks, Appl. Phys. Lett., 2000, 77, 2961.

39. L. Wu, L. Feng, J. Ren, X. Qu Biosensors and Bioelectronics 2012, 34, 57-62

40. J. K. Choi, G. Sargsyan, A.M. Olive and M. Balaz Chem. Eur. J., 2013, 19, 2515-2522. 
41. X.L. Zhang, Z.Y. Qin, X. Liu, B.L. Liang, N. Liu, Z. Zhou, M.F. Zhu, J. Mater. Chem. A $2013,1,10327-10333$

42. S. Banerjia, W. Penga, Y.-C. Kimb, N. Menegazzob, K.S. Booksh Sensors and Actuators B: Chemical 2010, 147, 255-262

43. K. Haupt, A.V. Linares, M. Bompart and T. S B. Bernadette, Topics in Current Chemistry, 2012, 325, 1-28. 


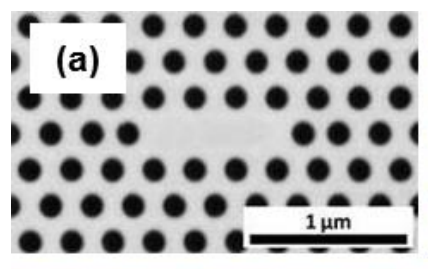

(d) (b)
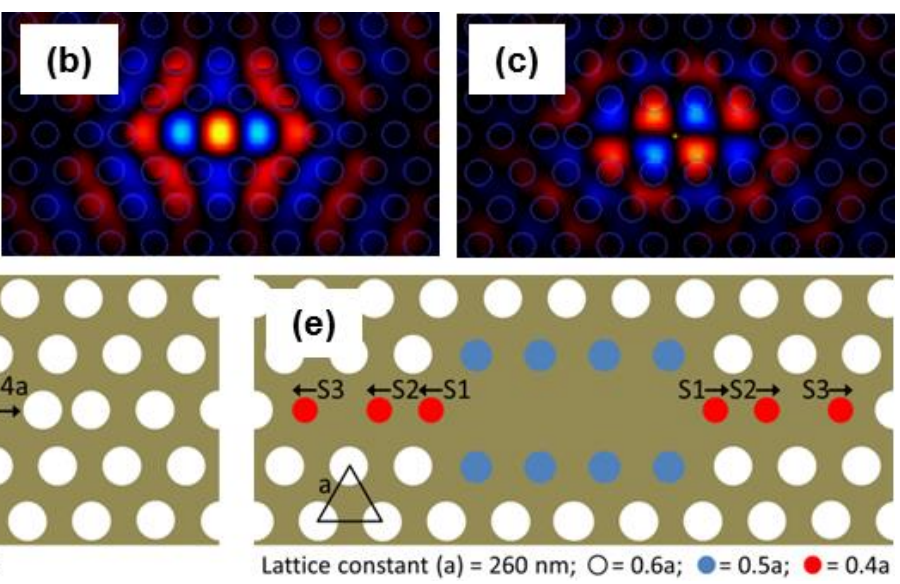

Figure 1: (a) SEM image of an L3 nanocavity fabricated into a $200 \mathrm{~nm}$ thick SiN membrane. Parts (b) \& (c) show an FDTD simulation of the $\mathrm{E}_{\mathrm{x}}$ and $\mathrm{E}_{\mathrm{z}}$ fields confined within the cavity respectively. Parts (d) \& (e) show a schematic of the two types of L3 nanocavities used in these experiments (described in the text as cavity (1) and cavity (2) respectively). 


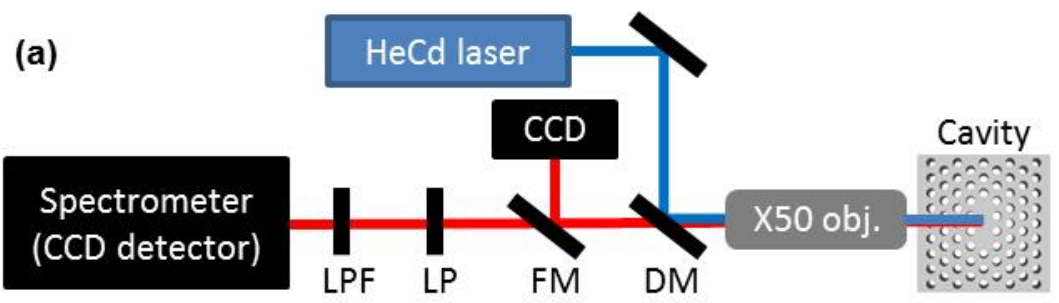

$\mathrm{LPF}=$ Long pass filter; $\mathrm{LP}=$ Linear polariser; FM = Flip mirror; $\mathrm{DM}=$ Dichroic mirror

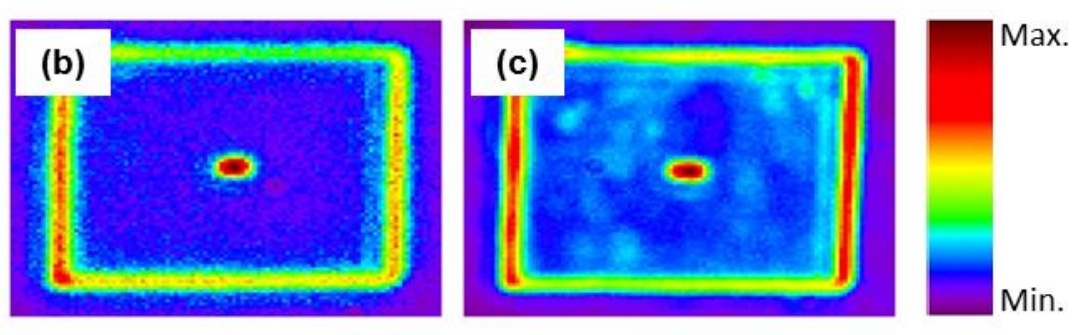

Figure 2: (a) Schematic representation of the experimental setup used in this work. Parts (b) \& (c) show PL intensity images of the PC and nanocavity before and after coating with a number of Lumogen-Red monolayers. Here, the colour-scale of both images are normalized to the peak emission intensity. 


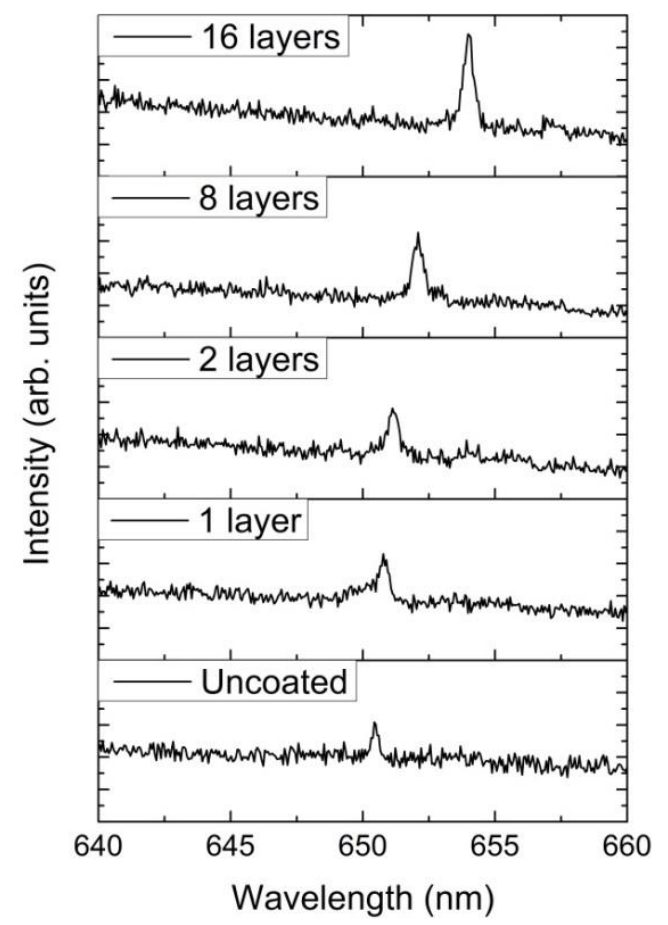

Figure 3: PL spectra for a Lumogen Red coated nanocavity as a function of the number of LS deposited monolayers. 

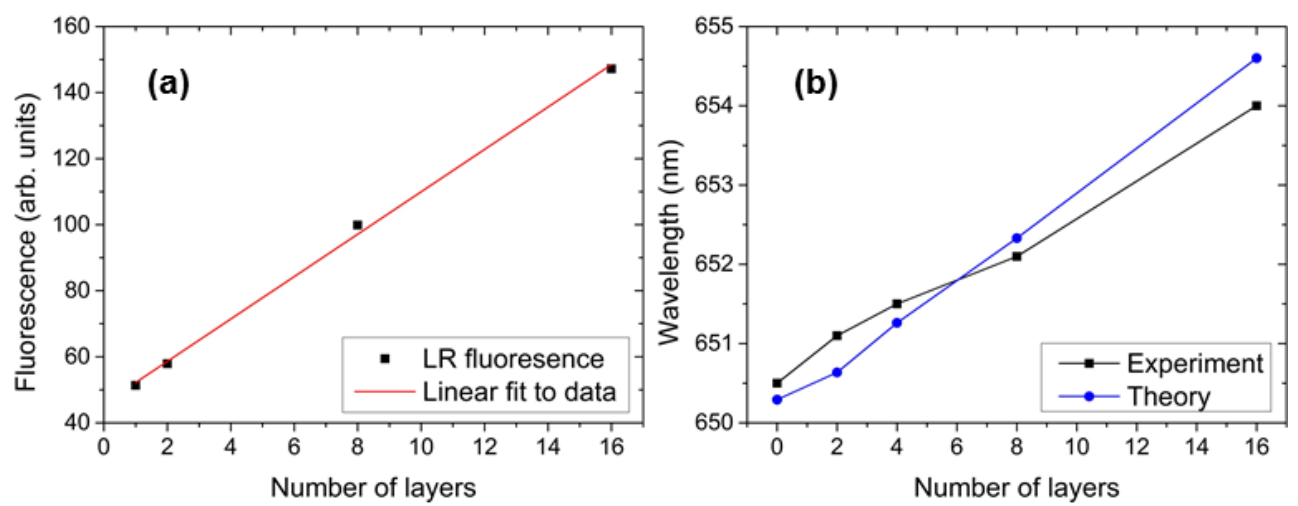

Figure 4: Part (a) shows the relative fluorescence intensity of the Lumogen Red (LR) emitted via the fundamental cavity mode cavity mode as a function of the number of molecular monolayers deposited onto the cavity surface. Part (b) shows the measured wavelength of the fundamental cavity mode of a nanocavity coated with Lumogen Red as a function of number of deposited monolayers. Data is compared with the results of an FDTD calculation plotted on the same wavelength scale. 
(a)

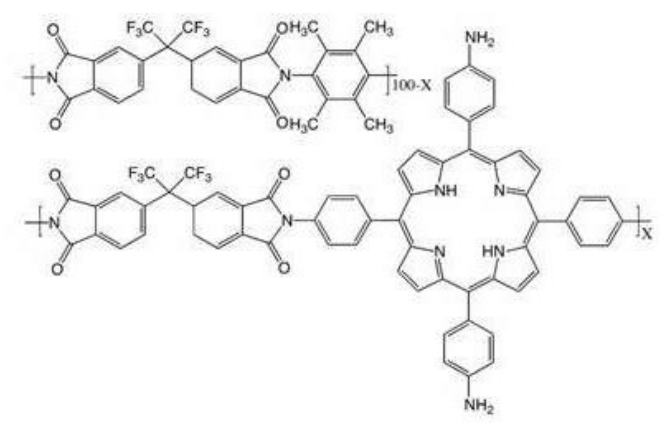

(c)

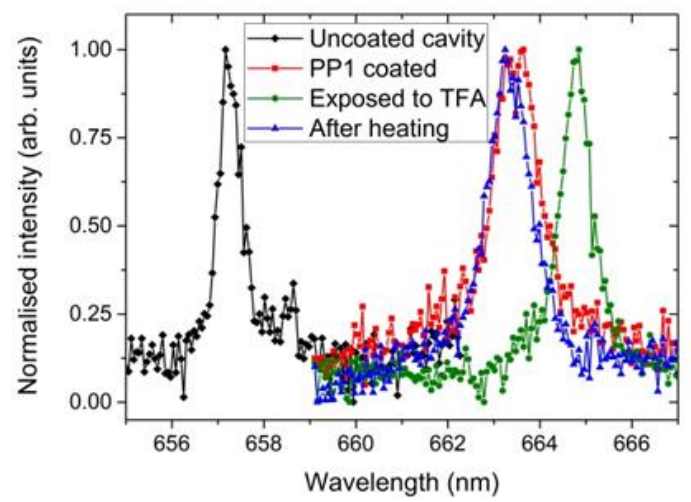

(b)
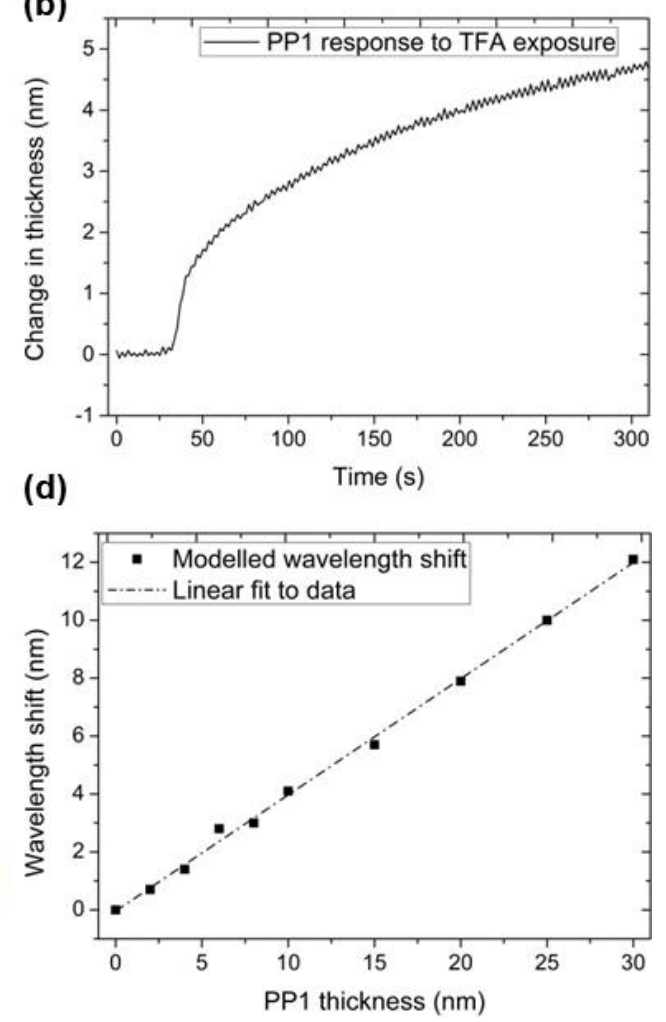

Figure 5: Part (a) shows the chemical structure of the PP1 polymer. Part (b) shows the increase in thickness of a control PP1 layer deposited on a silicon wafer as it is exposed to a TFA vapour. Here, thickness is determined via spectroscopic ellipsometry. Part (c) shows the PL emission from a nanocavity that has been coated with PP1. Here, data is shown for a cavity both before and after TFA vapour exposure. Data is also shown for the vapour-exposed cavity after it had been heated to $70^{\circ} \mathrm{C}$ and then returned to room temperature. Part (d) shows the result of an FDTD model that describes the shift in the cavity mode wavelength as a function thickness of the PP1 surface-layer. 


\section{A chemical sensor based on a photonic-crystal L3 nanocavity defined in a silicon-nitride membrane}

Deasy, Kieran

Royal Society of Chemistry

Deasy et al. (2014) A chemical sensor based on a photonic-crystal L3 nanocavity defined in a silicon-nitride membrane, Journal of Materials Chemistry C, Vol. 2, Iss. 41, pp. 2-41

http://dx.doi.org/10.1039/C4TC01320D

Downloaded from Cranfield Library Services E-Repository 